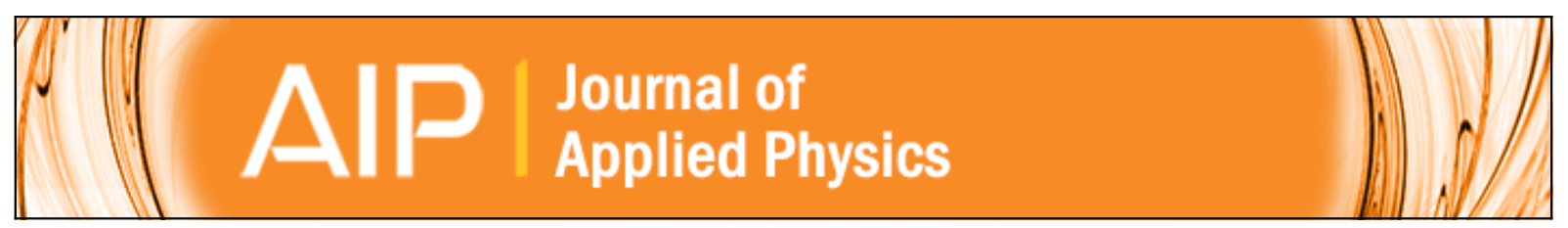

\title{
Droplet impact behavior on heated micro-patterned surfaces
}

Wenbin Zhang, Tongxu Yu, Jing Fan, Weijie Sun, and Zexian Cao

Citation: Journal of Applied Physics 119, 114901 (2016); doi: 10.1063/1.4943938

View online: http://dx.doi.org/10.1063/1.4943938

View Table of Contents: http://scitation.aip.org/content/aip/journal/jap/119/11?ver=pdfcov

Published by the AIP Publishing

\section{Articles you may be interested in}

Phase emerging from intramonolayer cycloaddition on micro-patterned monolayer

AIP Conf. Proc. 1502, 171 (2012); 10.1063/1.4769142

A study on the dynamic behaviors of water droplets impacting nanostructured surfaces

AIP Advances 1, 042139 (2011); 10.1063/1.3662046

Micro-patterned porous silicon using proton beam writing

AIP Conf. Proc. 866, 269 (2006); 10.1063/1.2401510

The critical temperature of dry impact for tiny droplet impinging on a heated surface

Phys. Fluids 12, 1622 (2000); 10.1063/1.870413

A study of diamond field emission using micro-patterned monolithic diamond tips with different $\mathrm{sp} 2$ contents Appl. Phys. Lett. 71, 3394 (1997); 10.1063/1.120346

\section{The new SR865 2 MHz Lock-In Amplifier ... \$7950}

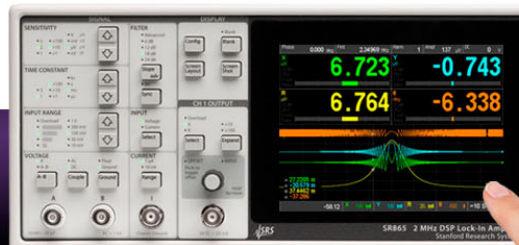

SRS Stanford Research Systems

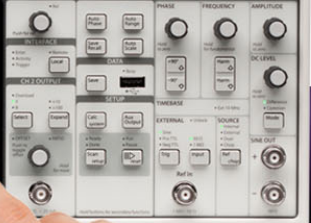

(॰)

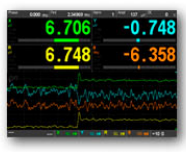

Chart recording

Intuitive front-panel operation

Touchscreen data display

Save data \& screen shots to USB flash drive

Embedded web server and iOS app

-Synch multiple SR865s via $10 \mathrm{MHz}$ timebase I/O

View results on a TV or monitor (HDMl output)

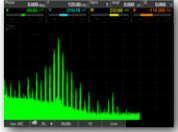

FFT displays

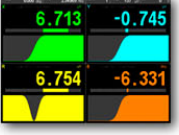

Trend analysis

$1 \mathrm{mHz}$ to $2 \mathrm{MHz}$

$2.5 \mathrm{nV} / \sqrt{ } \mathrm{Hz}$ input noise $1 \mu \mathrm{s}$ to $30 \mathrm{ks}$ time constants $1.25 \mathrm{MHz}$ data streaming rate

Sine out with DC offset

GPIB, RS-232, Ethernet \& USB 


\title{
Droplet impact behavior on heated micro-patterned surfaces
}

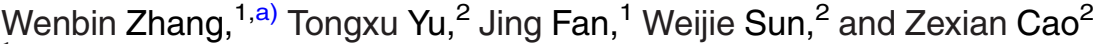 \\ ${ }^{1}$ Institute of Mechanics, Chinese Academy of Sciences, 100190 Beijing, China \\ ${ }^{2}$ Institute of Physics, Chinese Academy of Sciences, 100190 Beijing, China
}

(Received 30 November 2015; accepted 1 March 2016; published online 15 March 2016)

\begin{abstract}
Impact behavior of droplets on a surface is an intriguing research topic, and its control should be very useful in diverse industrial applications. We investigated the impact behavior of water droplets on the textured and chemically treated surface of silicon and obtained the impact mode map on the parameter plane subtended by the Weber number (up to 85) and temperature (up to $\left.320^{\circ} \mathrm{C}\right)$. The patterns comprise of micropillars $(14 \mu \mathrm{m}$ in height) in square lattice with a lattice constant of 10 and $20 \mu \mathrm{m}$, and the surface was further made superhydrophobic by coating with graphene nanosheets. Six distinct impact modes are identified. It was found that the impact mode map can be dramatically altered by modifying the texture and chemistry of the surface, and the observations are well explained with regard to heat transfer, vapor/bubble generation and vapor flow beneath the droplet. Instability in the droplet arising from the mismatch between vapor generation rate and exhaust conditions is the dominant factor in determining the impact mode. Our results revealed more facts and features of the droplet impact phenomenon and can be very useful for target-oriented surface design towards precise control of droplet impact behavior on heated substrates. C 2016 AIP Publishing LLC. [http://dx.doi.org/10.1063/1.4943938]
\end{abstract}

\section{INTRODUCTION}

Liquid droplet impacting onto a heated surface is a ubiquitous phenomenon which can be found in many industrial processes such as fuel injection for combustion, thermal spray coating, and spray cooling in various cooling systems. When the substrate's temperature is sufficiently high above the boiling point, the droplet can levitate itself over the substrate instead of experiencing a sudden boiling. A rapidly grown vapor layer completely separates the liquid from the heated surface. Due to the poor thermal conductivity of the vapor cushion, the heat flux from the substrate to the liquid dramatically decreases; consequently, the lifetime of the droplet will be much prolonged. This effect is well known as the Leidenfrost effect, ${ }^{1-4}$ and it plays an important role in situations where the behavior of droplets on heated substrate held at a temperature above the Leidenfrost temperature is concerned. The Leidenfrost state is often considered as a perfect superhydrophobic state. ${ }^{5}$ As the impacting droplet is not in direct contact with the substrate, it can move without friction on the substrate. For this reason, many studies have tried to control the movement of the Leidenfrost droplets by designing particular micropatterns. ${ }^{6-9}$ Other studies have dealt with the potential usage of the vapor layer to reduce the drag on a hot object moving in a liquid. ${ }^{10,11}$ It was also suggested that the Leidenfrost droplets would be used as a confining geometry to study colloidal packing. ${ }^{12}$ On the other hand, in the Leidenfrost state, the vapor layer blocks the heat transfer from the hot solid to the liquid, so the efficiency of the cooling system is drastically reduced, and the substrate can become overheated, such as those experienced in the Fukushima disaster.

\footnotetext{
a) Author to whom correspondence should be addressed. Electronic mail: wbzhang@iphy.ac.cn
}

The droplet impact behavior, being quite complicated, is anticipated to be very different above and below the Leidenfrost temperature. For a given surface, the droplet impacting behavior is determined by factors such as its thermal conductivity, the vapor/bubble generation rate and distribution, and the lateral flow and stability of the vapor, etc. Many experimental efforts were devoted to finding a way to precisely control the impact behavior, in particular, to alter the Leidenfrost temperature. Towards this end, various strategies have been adopted, including modifying the substrate surface, changing the surface tension of the liquid as well as the manner of droplet deposition, or applying an electric field between the droplet and the heated surface. ${ }^{12}$ Some studies claim that surface roughness is an important feature that can lead to an increase in Leidenfrost temperature, ${ }^{13-16}$ while others show a reversed trend. ${ }^{17,18}$ In recent years, micropatterned surfaces have also drawn much attention. ${ }^{19,20}$ Recently, it was demonstrated that surface texturing and chemical treatment can produce droplet-surface interactions that prohibit liquid and freezing droplet retention on surface. ${ }^{21}$ Although these studies have shed some light on the various factors that may play a role in determining the droplet impact behavior, the droplet impact mode map, something similar to the $\mathrm{p}-\mathrm{V}$ phase diagrams in thermodynamics, with regards to the governing parameters such as Weber number of droplet, the actual temperature of the droplet in case for heated substrate, the features of the substrate surface and so forth, is far from complete or well established. Such impact mode maps, which are governed by parameters such as the Weber number of droplets, the latter's actual temperature, the substrate surface features, and so forth, are expected to demonstrate explicitly the effectiveness of the various control factors in altering the impact behavior. Moreover, other impact modes may be found under different combinations of conditions. 
In the current article, we present our investigations on the impact behavior of droplets on heated silicon wafers, of which the surfaces were etched with a pattern of micrometer-sized pillars arranged in a square lattice, and some were further coated with graphene nanosheets to make them superhydrophobic. Six distinct impact modes were recognized, and the impact mode map on the parameter plane subtended by the Weber number and temperature has been obtained for different surfaces. The observed mode maps can be well explained with regard to the heat transfer, vapor generation (and bubble generation when available), and lateral flow of the vapor. The impact mode map can be dramatically altered by modifying the texture and chemistry of the surface, such that the domains for distinct impact modes to appear may be reshuffled and significantly deformed. The instability of the droplet caused by the incompatible vapor generation rate and exhaust condition is found to be the dominant factor in determining the impact mode.

\section{EXPERIMENTAL}

All the experiments were performed with de-ionized water (Millipore water) with a density of $\rho_{1}=998 \mathrm{~kg} / \mathrm{m}^{3}$, surface tension of $\gamma=72 \mathrm{mN} / \mathrm{m}$, and kinematic viscosity of $\nu=1.004 \times 10^{-6} \mathrm{~m}^{2} / \mathrm{s}$ at room temperature. The individual water droplets were prepared by a custom-designed syringe with a fine needle. The initial diameter of the droplet when detaching from the needle was kept at $\mathrm{D}_{0} \sim 1.9 \mathrm{~mm}$, which is considerably smaller than the capillary length for water, $\sqrt{\gamma / \rho_{l} g} \sim 2.71 \mathrm{~mm}$ (at room temperature), thus their shape can be regarded as spherical due to the dominating surface tension. The Weber number, defined as $W e=\rho_{l} V^{2} D_{0} / \gamma$, is a dimensionless quantity that characterizes the ratio of kinetic energy to the surface energy and consequently specifies the relative importance of these two types of energy; it is a pertinent parameter governing the impact process of droplets. ${ }^{22} \mathrm{In}$ the current work, the impact velocity was controlled by adjusting the release height $\mathrm{H}$ between 2 and $14 \mathrm{~cm}$. The corresponding Weber number calculated from $2 \rho_{l} g D_{0} H / \gamma$, derived by substituting $2 \mathrm{gH}$ for $\mathrm{V}^{2}$ in the definition, falls within the range from 12 to 85 . Here, the values of the density and surface tension at boiling point, namely, $958 \mathrm{~kg} / \mathrm{m}^{3}$ and $59 \mathrm{mN} / \mathrm{m}$, respectively, are used. For comparison, experiments were performed on silicon wafers with a flat, textured, and/or chemically treated surface (as specified below). The silicon wafers were placed on an aluminum plate which can be heated up to $400{ }^{\circ} \mathrm{C}$ by four embedded cartridge heaters, and their surface temperature was monitored by a thermocouple. A high speed camera (Giga View, SVSI) was employed to record the boiling and bouncing processes of the impacting droplets from a side view, the frame rate was set at $2000 \mathrm{fps}$, with an exposure time of $80 \mu \mathrm{s}$.

The substrates with a micro-patterned surface were fabricated by reactive ion etching (PlasmaLab System 100, Oxford Instruments) of polished silicon wafers, in which $\mathrm{SF}_{6}$ and $\mathrm{O}_{2}$ were used as the etching gases, and a cryogenic temperature of $-110^{\circ} \mathrm{C}$ was maintained during the fabrication process. The patterns comprise of micropillars $(\sim 5.0 \mu \mathrm{m}$ in diameter and $14 \mu \mathrm{m}$ in height) arranged in a square lattice.
Furthermore, for some of the samples the surface was coated with $\sim 50 \mathrm{~nm}$ thick graphene sheets via hot filament chemical vapor deposition. ${ }^{23,24}$ Patterned surfaces with two different lattice constants, one is $10 \mu \mathrm{m}$ and the other is $20 \mu \mathrm{m}$, were tested. To facilitate discussion, the plain patterned substrates will be denoted as P10 and P20, while those with graphene coating accordingly as CP10 and CP20. The morphological and structural features of the micro-patterned samples were characterized by using scanning electron microscopy (SEM, FEI XL30) and are displayed in Fig. 1. The contact angle of the droplet was measured with a goniometer (Krüss DSA100) equipped with a dispensing needle.

\section{RESULTS AND DISCUSSION}

The impact process of a droplet depends on its velocity and size, which are incorporated into the Weber number, the temperature and also the morphology and chemistry of the substrate surface. In the landscape of Weber number and substrate temperature, six distinctly different impact modes for droplets falling onto a flat surface can be unambiguously recognized, namely, the wetting mode, the contact boiling mode, the transition mode, the breaking mode, the rebounding mode, and the rebounding with satellite mode, as illustrated in Fig. 2. The simple wetting mode occurs when the heating is incapable of causing immediate boiling of water (Fig. 2(a)). In this case, the droplet spreads outwards when coming into contact with the substrate; once a maximum spreading has been reached, it will pull back to assume the sessile profile with a constant contact angle. In the contact boiling mode, the droplet in good contact with the surface experiences a high-rate inflow of heat, and vapor bubbles grow from inside, causing disruption of the liquid surface and sometimes ejection of tiny droplets (Fig. 2(b)). For this,

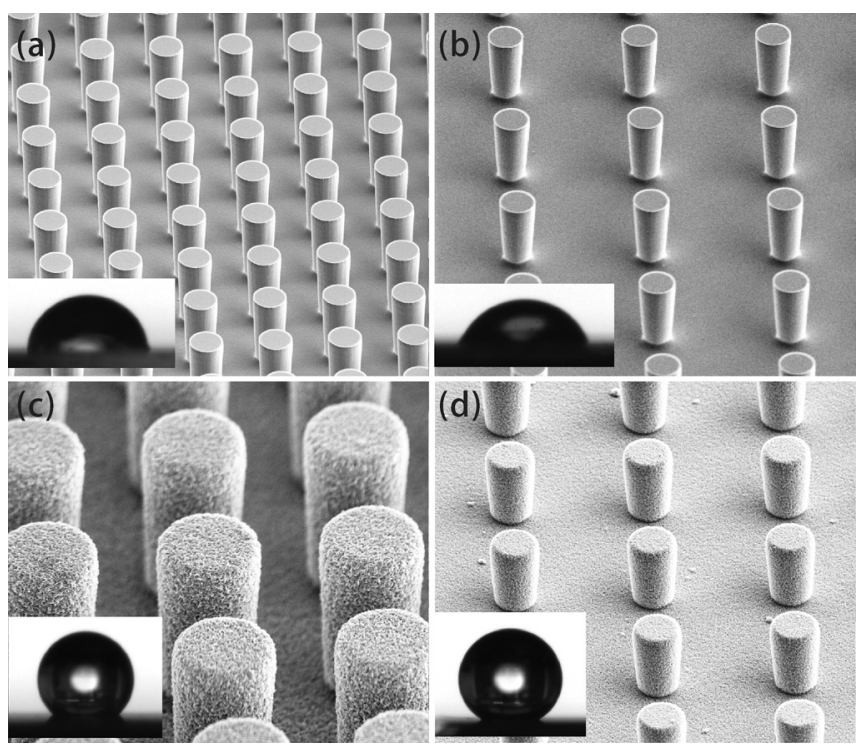

FIG. 1. SEM images of the patterned silicon substrates. The individual micropillars measure $5.0 \mu \mathrm{m}$ in diameter and $14 \mu \mathrm{m}$ in height, and the lattice constant for the square pattern is $10 \mu \mathrm{m}$ in (a) and $20 \mu \mathrm{m}$ in (b). In (c) and (d) displayed are, respectively, the patterned substrates in (a) and (b) now coated with a $\sim 50 \mathrm{~nm}$ thick graphene layer. Insets show the sessile water droplets on the corresponding substrates at room temperature, the contact angles are (a) $70^{\circ}$, (b) $62^{\circ}$, (c) $115^{\circ}$, and (d) $130^{\circ}$. 
(a)

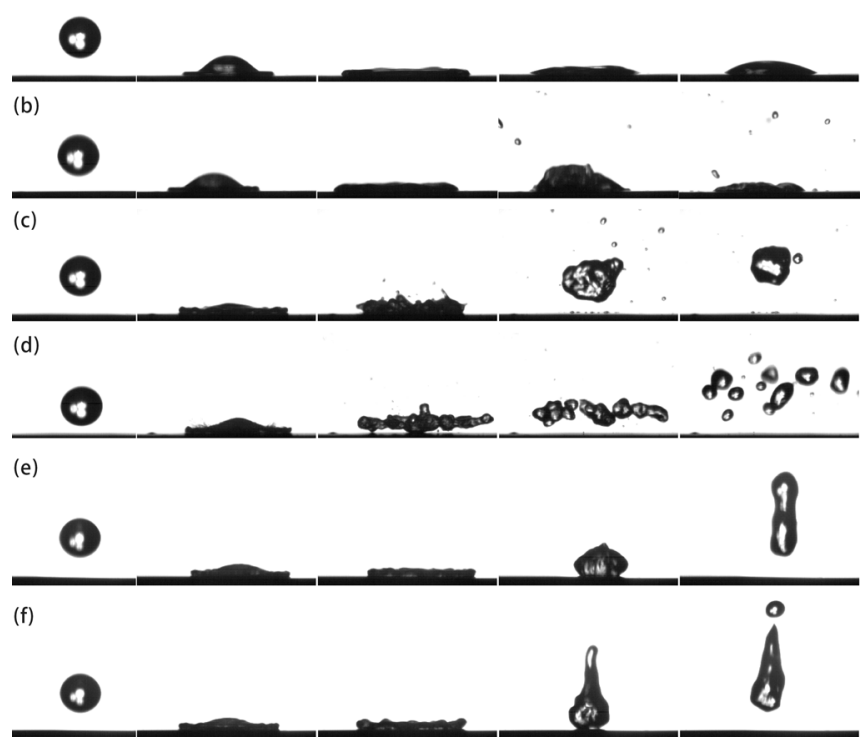

FIG. 2. Typical profile evolution of water droplets during impact on the heated flat silicon substrate for different Weber number. (a) Wetting mode at $\mathrm{We}=85, \mathrm{~T}=50^{\circ} \mathrm{C}$; (b) Contact boiling mode at $\mathrm{We}=85, \mathrm{~T}=110^{\circ} \mathrm{C}$; (c) Transition mode at $\mathrm{We}=85, \mathrm{~T}=150^{\circ} \mathrm{C}$; (d) Breaking mode at $\mathrm{We}=85$, $\mathrm{T}=210^{\circ} \mathrm{C}$; (e) Rebounding mode at $\mathrm{We}=66, \mathrm{~T}=270^{\circ} \mathrm{C}$ which appears above the Leidenfrost temperature of $\sim 260^{\circ} \mathrm{C}$; and (f) Rebounding with satellite mode at $\mathrm{We}=66, \mathrm{~T}=290^{\circ} \mathrm{C}$. Lapse time for the photo series: $5.0 \mathrm{~ms}$.

a sufficiently high heat transfer rate is required, which corresponds to a substrate temperature between 100 and $140^{\circ} \mathrm{C}$. When the substrate temperature rises to roughly $140^{\circ} \mathrm{C}$, the droplet can be finally elevated by vapor impulse, and this bouncing-off process is also accompanied by the sprinkling of tiny droplets. We denote this behavior as the transition mode (Fig. 2(c)). When the impact velocity is also raised, and correspondingly the Weber number, the droplet will be violently perturbed by the impact at the surface that it will break into several (usually less than 10) smaller droplets immediately after bouncing off the substrate, demonstrating a breaking mode (Fig. 2(d)). At temperatures above the socalled Leidenfrost temperature, when the falling droplet approaches the substrate, a vapor cushion beneath the droplet will be formed through liquid vaporization. This vapor cushion acts as a lubricant layer, which also reduces the energy dissipation during droplet spreading and recoiling. In this case, if the vapor layer is sufficiently stable, the impacting droplet can rebound back as a whole, becoming elongated in the process (Fig. 2(e)). This is the rebounding mode. However, capillary wave will be provoked, which will propagate along the liquid surface in such an elongated water column that it may cause the droplet to pinch off, generating a secondary droplet at the top (Fig. 2(f)). This is the so-called rebounding with satellite mode. The features presented in Fig. 2 partly reproduce the result of the work of Tran in 2012. ${ }^{25}$ Roughly speaking, Figs. 2(b) and 2(c) correspond to the contact boiling mode, while Figs. 2(e) and 2(f) correspond to the film boiling mode there. The breaking mode in Fig. 2(d) has not been mentioned together with the other impact modes in previous studies.

\section{A. Impact on flat surfaces}

The various kinds of droplet impact on a heated surface can be mapped on the parameter plane spanned by the Weber number, We, and temperature, $\mathrm{T}$, resulting in a "mode map" analogous to the $\mathrm{p}-\mathrm{V}$ phase diagram in thermodynamics. Figure 3 displays the mode map on the We-T plane, which shows regimes of distinct impact behaviors for water droplets falling on a flat silicon substrate. For $\mathrm{T} \leq 100^{\circ} \mathrm{C}$, the contact mode is observed across the given range of Weber number. When the temperature is over $110^{\circ} \mathrm{C}$ but below $140{ }^{\circ} \mathrm{C}$ (for larger Weber numbers, this temperature is a little lower), the contact boiling mode dominates; however, for temperatures over $140{ }^{\circ} \mathrm{C}$ or so, it shifts to the transition mode which appears in a narrow region, and, with increasing temperature, adjoins with rebounding/breaking mode at the smaller/larger Weber number side. The triple points separating the transition, the breaking and the rebounding modes lies roughly at the point of $\left(38,200^{\circ} \mathrm{C}\right)$. The region for the breaking mode is roughly a wedge, pointing towards the low Weber number direction. This domain looks similar to the "fingering boiling" regime described in the recent work of Khavari, derived from the bottom-view of the impacting droplets. ${ }^{26}$ There the "fingering boiling" droplets will break up into smaller droplets, they may refer to the same situation as the breaking mode here. The rebounding mode appears at the upper left part with respect to the breaking mode and terminates at the rebounding with satellite mode at the hightemperature end. Although the rebounding with satellite mode was observed, it is however intrinsically unstable, thus its observation in the current work suffers from an unsatisfactory reproducibility. The Leidenfrost temperature in this case is $\sim 170{ }^{\circ} \mathrm{C}$.

The mode map for droplet impacting on the flat surface in Fig. 3 is quite easy to understand. When a falling droplet comes into contact with the substrate, it begins to spread out laterally over the surface forming a liquid sheet. The area of

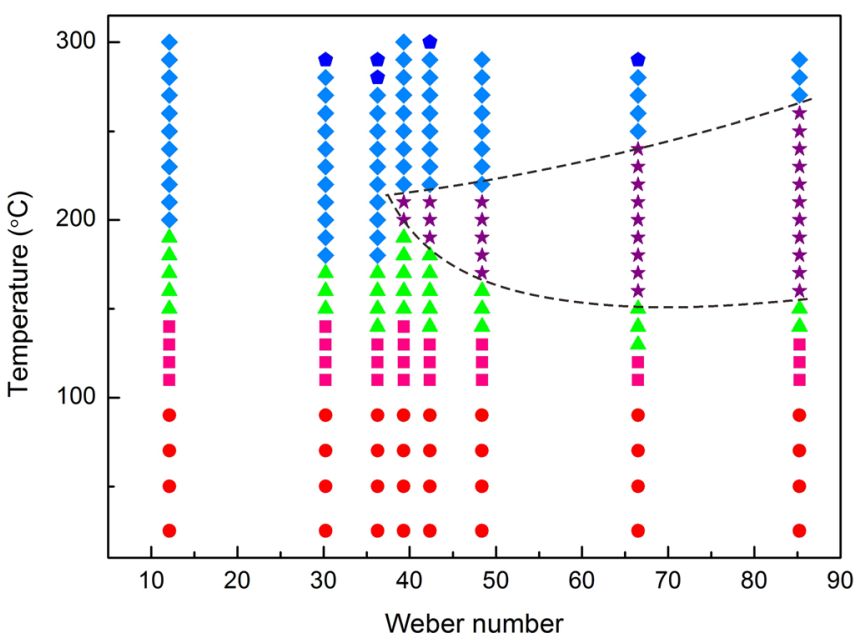

FIG. 3. Mode map on the We-T plane for the impact of water droplet on a flat silicon substrate. The various outcomes of impact behavior are marked with different symbols. Red circles: wetting mode; pink squares: contact boiling mode; green up triangles: transition mode; purple stars: breaking mode; blue diamonds: rebounding mode; dark blue pentagons: rebounding with satellite mode. 
its base expands by some factor, which increases with the Weber number since this is the process in which the kinetic energy transfers into surface energy. For small Weber number and low temperature, simple wetting mode occurs since the recoiling droplet can assume a stable cap shape with a constant contact angle to the substrate surface. However, on a heated surface, the heat transfer rate will be enhanced, and some vapor is generated underneath the droplet. In the case that the vapor layer between the extended droplet and the hot surface is unstable and the liquid sheet is in contact with the surface at sporadic places, bubbles (visible with high speed videos) can grow rapidly and burst out from the free surface, resulting in the breaking-up of the liquid sheet-this is the breaking mode (cf. Fig. 2(d)). If the substrate temperature is high enough that a thicker vapor cushion forms between the liquid and the surface immediately upon impact, this vapor layer is stable and the liquid is isolated from the heating surface. The droplet can enjoy the chance to retract due to capillary action, even causing the rebound of droplet. In this case, the droplet behaves as if it hit on a superhydrophobic surface, where similar rebounding mode was observed, and termed as the pancake rebound. ${ }^{27}$ As the droplet elongates, it can pinch off at the top, and eject a satellite droplet, this is a kind of Plateau-Rayleigh instability. These last two impacting modes refer to the so-called Leidenfrost state, ${ }^{1}$ and the onset of the rebound mode defines the Leidenfrost temperature. Generally speaking, the larger the Weber number, the higher a substrate temperature is required to initiate the rebounding mode. ${ }^{25}$ The rebounding droplet may experience a violently oscillating shape deformation, in which part of the energy is dissipated.

It seems that the availability and furthermore the stability of the vapor cushion are the key factors in determining the impact behavior of the droplets on a heated surface. The value of the thermal conductivity for water at the boiling point is $0.57 \mathrm{~W} / \mathrm{m} \cdot \mathrm{K}$ while for water vapor ${ }^{28}$ at the boiling point and under the atmospheric pressure it is only 0.016 $\mathrm{W} / \mathrm{m} \cdot \mathrm{K}$. If a stable vapor cushion blocks the direct contact between the droplet and the surface, the heat transfer rate will be drastically lowered, thus subsequently the droplet behaves according to its own dynamics. For a water droplet of $1.9 \mathrm{~mm}$ in diameter, the typical impact time is about $5 \mathrm{~ms}$, assuming a latent heat of evaporation of $\sim 2.26 \times 10^{6} \mathrm{~J} / \mathrm{kg}$ and a temperature gradient of $200 \mathrm{~K} / 10 \mu \mathrm{m}$, and in this period about $1.88 \times 10^{-6} \mathrm{mg}$ of water turns into vapor. But this mass of water vapor, if it is under ambient pressure, demands a space volume $\left(\sim 2.35 \mathrm{~mm}^{3}\right)$ a hundred times larger than that directly beneath the droplet $\left(\sim 0.028 \mathrm{~mm}^{3}\right)$. The flow of the escaping vapor is assuredly an important factor to cause instability. If the lateral flow is blocked, the large pressure of the vapor will be able to push up the droplet. Based on the work of Biance, ${ }^{3}$ the vapor layer supplied by evaporation from the bottom of the droplet flows laterally due to the droplet weight. For a stably levitated droplet, the vapor mass trapped in the vapor layer should be a constant, i.e., the continuous evaporation and lateral flow are mutually balanced. Thus, the thickness of the vapor layer can be expressed as $h \approx c\left(\frac{k_{V} \Delta T \mu_{V} \rho_{L} g}{L \rho_{V} \gamma^{2}}\right)^{1 / 3} D_{0}^{4 / 3}$, where $\mu_{V}$ is the dynamic viscosity of the air and $c$ is an adjustable coefficient. In the current work, the vapor layer thickness is estimated to be about $10 \mu \mathrm{m}$. With the $14 \mu \mathrm{m}$-high micropillars on the patterned surfaces, by means of which the heat transfer capability and the vapor flow are simultaneously altered, ${ }^{29}$ a drastic alteration of the mode map is anticipated.

\section{B. Impact on patterned surfaces}

For the four substrates with the patterned surface presented in Figs. 1(a)-1(d), denoted as P10, P20, CP10, and CP20, the individual pillars are exclusively $5 \mu \mathrm{m}$ in diameter and $14 \mu \mathrm{m}$ in height. The pillars form a regular square lattice, and the graphene coating for samples CP10 and CP20 looks quite uniform (Fig. 1). The contact angles of the water droplet are $70^{\circ}$ for $\mathrm{P} 10,62^{\circ}$ for $\mathrm{P} 20,115^{\circ}$ for $\mathrm{CP} 10$, and $130^{\circ}$ for CP20, showing that both the texture and the hydrophobic coating contribute, respectively, to raise the contact angle (for the flat silicon surface, the contact angle is $42^{\circ}$ ). When only micropillars are present as in samples P10 and P20, the contact angle is increased, yet the surface is still hydrophilic as the water droplet can penetrate into the interspace between the micropillars, i.e., the droplet contacts with the substrate in the Wenzel mode. By contrast, the graphenecoated surface is hydrophobic, so the water droplet there cannot penetrate into the interspace between the micropillars, i.e., the droplet contacts with the substrate in the CassieBaxter mode. ${ }^{30}$ Due to the existence of micropillar-spanned empty space in these substrates, the impact behavior of the droplet is very different.

Figure 4 shows the mode maps for droplet impacting on the four micro-patterned substrates referred to Fig. 1. Notably, Fig. 4(a) still bears resemblance to Fig. 3 for flat surface at low temperatures not far above $100^{\circ} \mathrm{C}$, as the substrate P10 is still hydrophilic and the impacting droplet on the substrate stays in the Wenzel mode. Below $100^{\circ} \mathrm{C}$ the wetting mode dominates. As the temperature increases above the water boiling point, vapor bubbles form on the hot surface quickly and burst through the droplet, generating sprinkling tiny droplets, i.e., there is now a shift to the contact boiling mode. The typical feature in Fig. 4(a) is that the transition mode (green triangles) only appears in a small region of low Weber number, and at $\mathrm{We}=30$, this temperature window is between $130^{\circ} \mathrm{C}$ and $180^{\circ} \mathrm{C}$. Remarkably, the region for the breaking mode expands outward significantly with increasing Weber number. It begins at $\mathrm{We}=30, \mathrm{~T}=180^{\circ} \mathrm{C}$, and borders directly with the contact boiling mode at the low temperature side. For the breaking mode at larger We, it persists as long as the temperature is over $130^{\circ} \mathrm{C}$. Even when the substrate temperature reaches $300^{\circ} \mathrm{C}$, the impact droplet cannot rebound back as a whole. This means the vapor layer formed beneath the impacting droplet on the micropillars with $5-\mu \mathrm{m}$ interspacing is very unstable, as now the micropillars piercing the droplet enhance the heat transfer from substrate, and the vapor pressure around the contact area is larger than elsewhere. Meanwhile, the smaller interspace between the micropillars is not wide enough for the rapidly growing vapor to escape without delay - the lateral flow is to some extent blocked. The high pressure of vapor, inhomogeneous and unstable, will easily break up the liquid sheet 

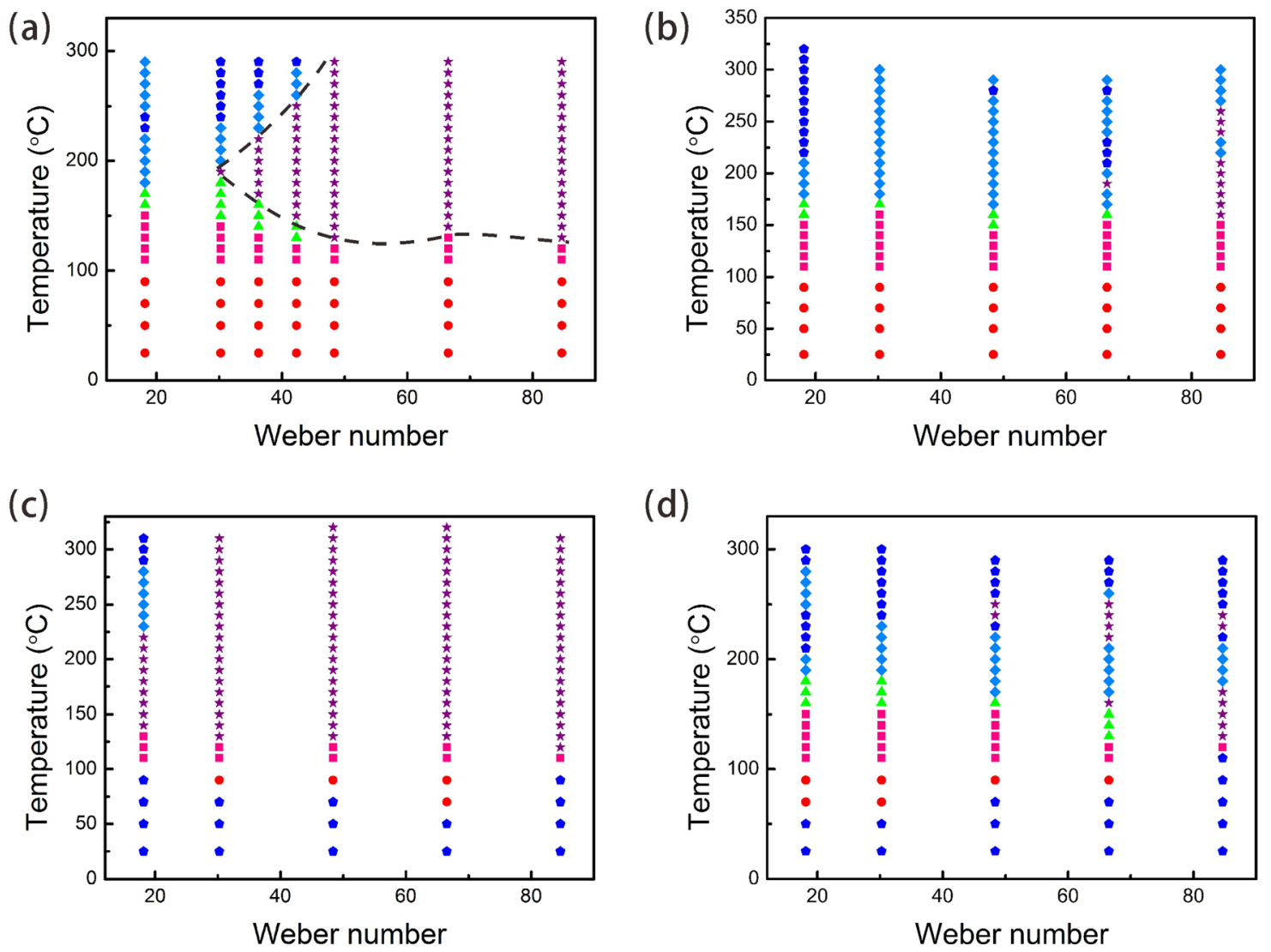

FIG. 4. Mode map on the We-T plane for the impact of water droplet on the patterned surfaces accordingly referred to Fig. 1. Red circles: wetting mode; pink squares: contact boiling mode; green up triangles: transition mode; purple stars: breaking mode; blue diamonds: rebounding mode; dark blue pentagons: rebounding with satellite mode.

from the spreading droplet. The higher the impact velocity, the thinner the liquid sheet formed, thus the easier it is for the breaking-up mode to occur. Clearly, in this case, the Leidenfrost temperature is significantly raised. This observation is consistent with previous studies. ${ }^{19}$

As to the impacting mode on the low We side, say, $\mathrm{We}=30$, the rebounding mode begins at $180^{\circ} \mathrm{C}$ (which can be regarded as the Leidenfrost temperature) and transits at $230^{\circ} \mathrm{C}$ to the rebounding with satellite mode. At $\mathrm{We}=18$, the rebounding mode, interrupted by rebounding with satellite mode at $230^{\circ} \mathrm{C}$ and $240^{\circ} \mathrm{C}$, extends to over $300^{\circ} \mathrm{C}$. But at $\mathrm{We}=30$, the rebounding mode turns firmly to the rebounding with satellite mode at $230^{\circ} \mathrm{C}$.

For substrate P20 where the micropillar interspacing is $15 \mu \mathrm{m}$, the low temperature part (below $150^{\circ} \mathrm{C}$ ) of the impact mode landscape in Fig. 4(b) is much like those for flat silicon wafer (Fig. 3) and for substrate P10 (Fig. 4(a)). The transition mode appears in a very narrow temperature window (at around $160^{\circ} \mathrm{C}$ ) and at a Weber number up to 66 , and the breaking mode appears only at sufficiently large Weber number (We $>66)$. Clearly, the large micropillar separation helps disperse the vapor, hence the breaking-up of droplet by vapor burst is suppressed. For $\mathrm{We}=18$, it has roughly the same Leidenfrost temperature as on P10 that the impacting droplet can rebound back at temperatures from $180^{\circ} \mathrm{C}$ up to $220^{\circ} \mathrm{C}$. It seems that the large separation of micropillars in this structure facilitates the formation of vapor layer demanded to levitate the impacting droplet to rebound back. From $220^{\circ} \mathrm{C}$ on, the rebounding droplet elongates to pinch off a satellite droplet. The high temperature part (above the Leidenfrost temperature) for Weber numbers between 30 and 66 is almost fully occupied by the rebounding mode, with the lower temperature border only shifted slightly down. The scarcely and random appearance of the rebounding with satellite mode at $\mathrm{We}=66$ is due to the successful trapping of vapor by the faster droplets. Obviously, the large micropillar spacing is favorable for the rebounding mode.

By comparing Figs. 4(a) and 4(b), we see that the detailed structure of the micropatterns can effectively modify the droplet impacting behavior thereupon. The role of the micropillars here is twofold. When the micropillar height is larger than the possible thickness of the vapor layer, direct contact of the liquid with the hot surface enhances the heat transfer rate, consequently raises the vapor generation rate which is one of the main factors that disrupt the profile of impacting droplet. On the other hand, the lateral flow of vapor beneath the droplet is to some extent suppressed by the micropillars, consequently high vapor pressure ensues so as to levitate or even pierce the droplet. In sample P10, more micropillars are available per unit area than in sample P20, thus more vapor is to be generated and maintained beneath the droplet. This explains why the mode map for sample P10 is dominated by the breaking mode. The Leidenfrost temperature for such a substrate will be much higher, for larger 
Weber numbers it is more than $300^{\circ} \mathrm{C}$ as estimated from the current work.

Surface wettability is another important factor in determining the droplet impact behavior. The presence of graphene nanosheets on the micropillars dramatically enhances the hydrophobicity of the patterned surfaces. When a water droplet is deposited on these surfaces, water does not penetrate into the interspacing of the micropillars, but held in the Cassie state. On the water repellent surface of CP10 and CP20, the impacting droplet is prone to rebounding back, which is consistent with the observation that on a superhydrophobic surface of microcavity lattice, the rebounding mode governs the low We $(<120)$ region. ${ }^{31}$ In fact, due to the hydrophobic nature of the surface together with easy air trapping, the rebounding droplets are more likely to pinch off at the top.

Unlike P10 and P20, for substrate CP10 the low temperature $\left(\leqslant 100^{\circ} \mathrm{C}\right)$ region is governed by the rebounding with satellite mode. Prior to pinching-off, the droplet suffers an elongation by a factor of 1.6-2.9. For medium Weber numbers $(\mathrm{We}=30-66)$ at temperatures around $90^{\circ} \mathrm{C}$, it reassumes the wetting mode. Via the contact boiling mode in a narrow window at a slightly elevated temperature above boiling point it enters the breaking mode. Roughly speaking, the map for $\mathrm{T}>120^{\circ} \mathrm{C}$ is fully occupied by the breaking mode, that only for $\mathrm{We}=18$ does it revert to the rebounding mode at $\mathrm{T}>230^{\circ} \mathrm{C}$, and to the rebounding with satellite mode at $\mathrm{T}>280^{\circ} \mathrm{C}$ (Fig. 4(c)). The transition mode is absent in this sample. Noticeably, the presence of graphene nanosheets promotes the local pressure in the superheated contacting area, as bubbles nucleate there more easily and grow rapidly. When the vapor expansion velocity is larger than the critical velocity for the initiation of the Kelvin-Helmholtz instability which is held responsible for splashing under various conditions, ${ }^{32}$ the vapor liquid interface will be disrupted to initiate the break-up of the thin extending liquid sheet, even for low Weber numbers so long as the temperature is sufficiently high. The Leidenfrost temperature for substrate
CP10 can be very high; in fact in Fig. 4(c) both the rebounding and rebounding with satellite modes at high temperatures only appear regularly for $\mathrm{We}=18$.

For substrate CP20, the situation is different from the other three cases. The landscape of the impact behavior becomes complicated and pierced (Fig. 4(d)). At low temperatures (roughly $<100{ }^{\circ} \mathrm{C}$ ) it is the rebounding with satellite mode which gradually transforms to the wetting mode especially at smaller Weber number as now the liquid has a smaller surface tension. The contact boiling mode occupies a large area especially at smaller Weber numbers, and it extends to the temperature of $150^{\circ} \mathrm{C}$. Notably, the breaking mode appears in two regions separated by the rebounding mode. For the lower region with $\mathrm{T} \leq 170^{\circ} \mathrm{C}$, the Weber numbers are 66 and 85 . The upper region for the breaking mode appears at $\mathrm{T} \sim 250^{\circ} \mathrm{C}$, and the Weber number can be as small as 48 . The rebounding mode is terminated by the rebounding with satellite mode at the high-temperature side, but for $\mathrm{We}=18$, the rebounding mode and rebounding with satellite mode appear alternatively. In this substrate CP20, although the contact between graphene nanosheets and the liquid can enhance heterogeneous nucleation bubbles, the vapor can be conducted away via lateral flow, so that the vapor layer can be stable enough to levitate the impacting droplet. This is why the breaking mode is less likely to occur. Yet the vapor pressure beneath the droplet is usually very large at high temperatures $\left(\mathrm{T} \geq 170^{\circ} \mathrm{C}\right)$, thus as the impacting droplet retracts, the vapor cushion will give the bouncing droplet a larger impetus, which makes it deform more violently along its length and become more likely to pinch off to eject a satellite droplet. This explains why the high temperature region for $\mathrm{CP} 20$ is dominated by the rebounding and rebounding with satellite modes.

The impacting behavior above the Leidenfrost temperature is of particular interest and deserves more attention. In this case, during the early stages of impact, the droplet will spread over the substrate to form a liquid sheet and then retract. In Fig. 5(a), we present as an example a plot of $D / D_{0}$ (a)

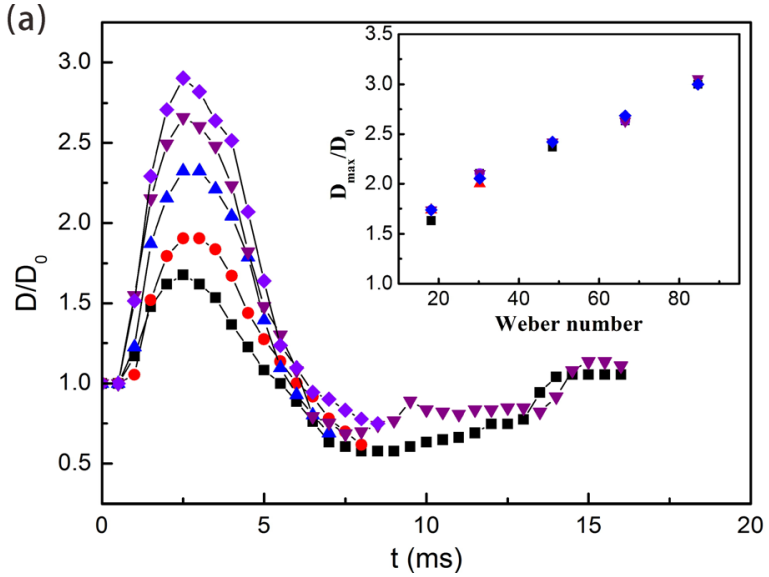

(b)

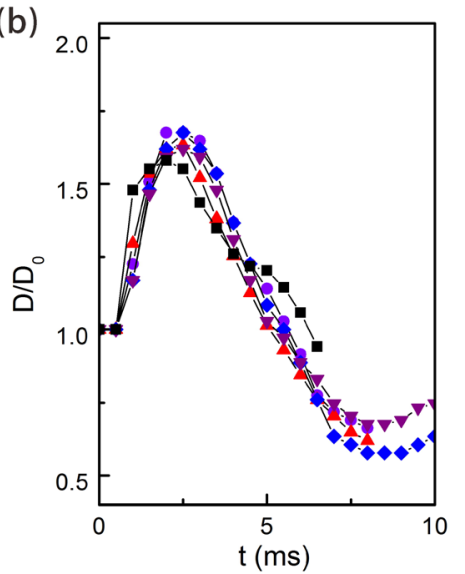

FIG. 5. Time evolution of the base diameter D normalized against the initial value $\mathrm{D}_{0}$ to illustrate the deformation of the droplets in the course of impacting. Results in (a) were obtained at $300{ }^{\circ} \mathrm{C}$ on the substrate CP20 referred to Fig. 1(d). The Weber number for the impacting droplet is 18 (square), 30 (circle), 48 (up triangle), 66 (down triangle), and 85 (diamond), respectively. Inset shows the spreading factor $D_{\max } / D_{0}$ versus Weber number obtained on flat substrate and on the four differently patterned substrates referred to Fig. 1, which are essentially indistinguishable. (b) illustrates the results obtained with a Weber number of 18 and at $300^{\circ} \mathrm{C}$ on different substrates: flat surface (square); P10 (circle); CP10 (up triangle); P20 (down triangle); and CP20 (diamond). 


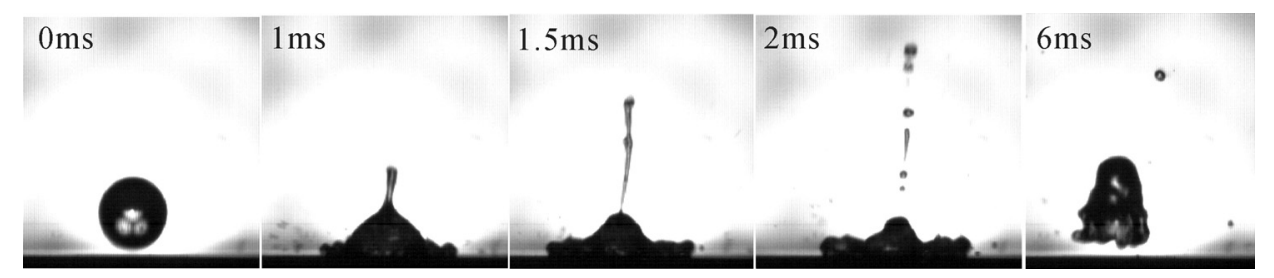

FIG. 6. Image sequence of an impacting water droplet showing jet ejection on the sample CP10 referred to Fig. 1(c). Weber number: 48 ; temperature: $170^{\circ} \mathrm{C}$.

as a function of time obtained on $\mathrm{CP} 20$ at $300^{\circ} \mathrm{C}$. The maximum spreading diameter $D_{m}$ increases linearly as the squared Weber number. Since the Weber number is proportional to the kinetic energy available to deform the droplet, and the maximum diameter of the disk that the latter can assume is limited by the total surface energy. So long as it is above the Leidenfrost temperature, the spreading of the droplet is only slightly altered by the surface condition of the substrates. For $\mathrm{We}=18$, the spreading factor $D_{\max } / D_{O}$ is about 1.74 on the patterned surfaces, and about 1.63 on a flat surface (Fig. 5(b)). As the Weber number increases to 85, the spreading factors all increase to about 3.0, yet still show very small difference. As can be anticipated, the spreading factor is also independent of the surface temperature.

The breaking mode for droplet impacting on the patterned hydrophobic substrates also deserves special attention, there often an upward water jet emerges at the center of the droplet. ${ }^{25}$ Figure 6 shows a series of images taken during the impact of a droplet on CP10. Shortly after the droplet comes into contact with the substrate, a jet is formed and ejected from the center of the spreading droplet. A possible explanation for this is that the vapor bubbles generated beneath the droplet apply a huge pressure to the liquid, causing the liquid in the center to shoot out. When the micropillars come into direct contact with the liquid surface at the droplet center, a bubble is formed immediately on the tip of the micropillars, and it grows quickly as the water droplet spreads. In the case that the outflow of the vapor is inhibited by the spreading droplet and the micropillars, the huge pressure inside the bubble is released by the ejection of the upward jet.

\section{CONCLUSIONS}

In this work, we have investigated the impact behavior of water droplets on heated micro-patterned surfaces. Six distinct impacting modes are identified and applied to characterize the impacting mode map on the plane subtended by the Weber number and temperature. By introducing micropillars of various separations and modifying the wettability of the surface with graphene coating, impact mode maps of essentially different features can be realized, which confirms the feasibility of impact mode control by surface design. A micropatterned hydrophobic surface with micropillars penetrating the vapor layer implies a raised Leidenfrost temperature, and once a stable vapor layer can be formed beneath the impacting droplet, the impact behavior is independent of either the detailed morphology of the surface, or the temperature. Remarkably, the instability of the droplet caused by the incompatible vapor generation rate and exhaust condition is found to be the dominant factor in determining those breaking modes. Our results reveal more facts and features of the droplet impact phenomenon, they can be very helpful for target-oriented surface design for the precise control of droplet impact on heated substrates confronted in various industrial applications.

\section{ACKNOWLEDGMENTS}

This work was financially supported by the National Basic Research Program of China Grant No. 2012CB933002, by the Natural Science Foundation of China Grant Nos. 11290161, 51172272, and 11474335, and by the Knowledge Innovation Project of Chinese Academy of Sciences Grant No. KJZD-EW-M03.

${ }^{1}$ S. Chandra and C. T. Avedisian, Proc. R. Soc. London, Ser. A 432, 13 (1991).

${ }^{2}$ D. Quere, Annu. Rev. Fluid Mech. 45, 197 (2013).

${ }^{3}$ A. L. Biance, C. Clanet, and D. Quere, Phys. Fluids 15, 1632 (2003).

${ }^{4}$ F. Celestini, T. Frisch, and Y. Pomeau, Soft Matter 9, 9535 (2013).

${ }^{5}$ D. Quere, Nat. Mater. 11, 915 (2012).

${ }^{6}$ H. Linke, B. J. Aleman, L. D. Melling, M. J. Taormina, M. J. Francis, C. C. Dow-Hygelund, V. Narayanan, R. P. Taylor, and A. Stout, Phys. Rev. Lett. 96, 154502 (2006).

${ }^{7}$ G. Lagubeau, M. L. Merrer, C. Clanet, and D. Quere, Nat. Phys. 7, 395 (2011).

${ }^{8}$ T. Baier, G. Dupeux, S. Herbert, S. Hardt, and D. Quere, Phys. Rev. E 87, 021001 (2013).

${ }^{9}$ G. Liu, L. Fu, A. V. Rode, and V. S. J. Craig, Langmuir 27, 2595 (2011).

${ }^{10}$ I. U. Vakarelski, J. O. Marston, D. Y. C. Chan, and S. T. Thoroddsen, Phys. Rev. Lett. 106, 214501 (2011).

${ }^{11}$ I. U. Vakarelski, N. A. Patankar, J. O. Marston, D. Y. C. Chan, and S. T. Thoroddsen, Nature 489, 274 (2012).

${ }^{12}$ C. H. Lim, H. Kang, and S. H. Kim, Langmuir 30, 8350 (2014).

${ }^{13}$ F. Celestini and G. Kirstetter, Soft Matter 8, 5992 (2012).

${ }^{14}$ H. Kim, B. Truong, J. Buongiorno, and L. W. Hu, Appl. Phys. Lett. 98, 083121 (2011).

${ }^{15}$ C. M. Weickgenannt, Y. Zhang, S. Sinha-Ray, I. V. Roisman, T. Gambaryan-Roisman, C. Tropea, and A. L. Yarin, Phys. Rev. E 84, 036310 (2011).

${ }^{16}$ H. Nair, H. J. Staat, T. Tran, A. van Houselt, A. Prosperetti, D. Lohse, and C. Sun, Soft Matter 10, 2102 (2014).

${ }^{17}$ D. A. del Cerro, A. G. Marin, G. Romer, B. Pathiraj, D. Lohse, and A. J. H. in't Veld, Langmuir 28, 15106 (2012).

${ }^{18}$ T. Tran, H. J. J. Staat, A. Susarrey-Arce, T. C. Foertsch, A. van Houselt, H. Gardeniers, A. Prosperetti, D. Lohse, and C. Sun, Soft Matter 9, 3272 (2013).

${ }^{19}$ H. Kwon, J. C. Bird, and K. K. Varanasi, Appl. Phys. Lett. 103, 201601 (2013).

${ }^{20}$ K. H. Chu, Y. S. Joung, R. Enright, C. R. Buie, and E. N. Wang, Appl. Phys. Lett. 102, 151602 (2013).

${ }^{21}$ T. M. Schutzius, S. Jung, T. Maitra, G. Graeber, M. Köhme, and D. Poulikakos, Nature 527, 82 (2015).

${ }^{22}$ A. L. Yarin, Annu. Rev. Fluid Mech. 38, 159 (2006).

${ }^{23}$ S. Tian, X. Xia, W. Sun, W. Li, J. Li, and C. Gu, Nanotechnology 22, 395301 (2011).

${ }^{24}$ S. Tian, L. Li, W. Sun, X. Xia, D. Han, J. Li, and C. Gu, Sci. Rep. 2, 511 (2012). 
${ }^{25}$ T. Tran, H. J. J. Staat, A. Prosperetti, C. Sun, and D. Lohse, Phys. Rev. Lett. 108, 036101 (2012).

${ }^{26}$ M. Khavari, C. Sun, D. Lohsec, and T. Tran, Soft Matter 11, 3298 (2015).

${ }^{27}$ Y. Liu, L. Moevius, X. Xu, T. Qian, J. M. Yeomans, and Z. Wang, Nat. Phys. 10, 515 (2014).

${ }^{28}$ online database Engineering ToolBox at http://www.engineeringtoolbox.com/thermal-conductivity-d_429.html.
${ }^{29}$ E. A. Silk, J. Kim, and K. Kiger, Int. J. Heat Mass Transfer 49, 4910 (2006).

${ }^{30}$ D. Quere, Annu. Rev. Mater. Res. 38, 71 (2008).

${ }^{31}$ H. Kim, U. Park, C. Lee, H. Kim, M. H. Kim, and J. Kim, Appl. Phys. Lett. 104, 161608 (2014).

${ }^{32}$ Y. Liu, P. Tan, and L. Xu, Proc. Natl. Acad. Sci. U. S. A. 112, 3280 (2015). 